CHALMERS GÖTEBORG UNIVERSITY

PREPRINT 2006:24

\title{
On a Certain Exponential Inequality for Gaussian Processes
}

\section{CHRISTER BORELL}

Department of Mathematical Sciences

Division of Mathematics

CHALMERS UNIVERSITY OF TECHNOLOGY

GÖTEBORG UNIVERSITY

Göteborg Sweden 2006 

Preprint 2006:24

\title{
On a Certain Exponential Inequality for Gaussian Processes
}

\author{
Christer Borell
}

CHALMERS $\mid$ GÖTEBORG UNIVERSITY
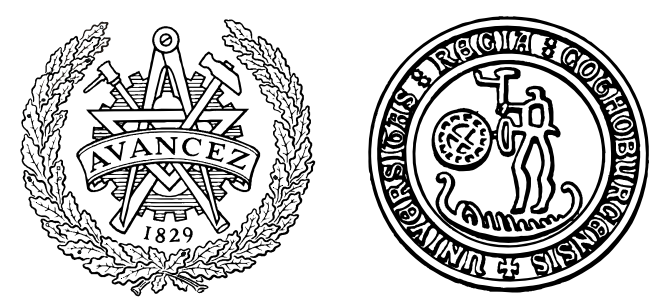

Department of Mathematical Sciences

Division of Mathematics

Chalmers University of Technology and Göteborg University

SE-412 96 Göteborg, Sweden

Göteborg, September 2006 
Preprint 2006:24

ISSN 1652-9715

Matematiska vetenskaper

Göteborg 2006 


\section{ON A CERTAIN EXPONENTIAL INEQUALITY FOR GAUSSIAN PROCESSES}

Christer Borell

School of Mathematical Sciences, Chalmers University of Technology and Göteborg University, S-412 96 Göteborg, Sweden

e-mail: borell@math.chalmers.se

\section{Abstract}

If $X=\left(X_{j}\right)_{j=1}^{m}$ is a zero-mean Gaussian process and $\sigma_{j}=\left(E\left[X_{j}^{2}\right]\right)^{1 / 2}$, $j=1, \ldots, m$, Tsirel'son (1985, Theory Probab. Appl. 30, 820-828) and more explicit Vitale (1996, Ann. Prob. 24, 2172-2178, and 1999, Contemp. Math. 234, 209-212) applied results from Brunn-Minkowski theory to show that $X$ satisfies the following inequality:

$$
E\left[\exp \left(\max _{1 \leq j \leq m}\left(X_{j}-\frac{\sigma_{j}^{2}}{2}\right)\right)\right] \leq \exp \left(E\left[\max _{1 \leq j \leq m} X_{j}\right]\right) .
$$

In this paper a more general inequality will be derived using a certain representation formula for Gaussian integrals. In particular, it also follows that

$$
E\left[\exp \left(\min _{1 \leq j \leq m}\left(X_{j}-\frac{\sigma_{j}^{2}}{2}\right)\right)\right] \leq \exp \left(E\left[\min _{1 \leq j \leq m} X_{j}\right]\right) .
$$

At the very end of the article certain option prices in the Black-Scholes and Bachelier models are compared.

Keywords: Gaussian processes; Brownian motion; Exponential inequality; Option pricing

\section{Introduction}

It is well known that methods from diffusion theory have often been useful in proving geometric inequalities of Gaussian processes. In this short note we will exhibit a new example. 
Throughout the paper, if not otherwise stated $m$ is a fixed positive integer, $X=\left(X_{j}\right)_{j=1}^{m}$ denotes a zero-mean Gaussian process, and $\sigma_{j}=\left(E\left[X_{j}^{2}\right]\right)^{1 / 2}$, $j=1, \ldots, m$.

The Alexandrov-Fenchel inequality and connections between mixed volumes and Gaussian processes have led to the following exponential inequality:

$$
E\left[\exp \left(\max _{1 \leq j \leq m}\left(X_{j}-\frac{\sigma_{j}^{2}}{2}\right)\right)\right] \leq \exp \left(E\left[\max _{1 \leq j \leq m} X_{j}\right]\right) .
$$

The inequality (1.1) is explicit in Vitale (1996) and is equivalent to Corollary 1 in Tsirel'son (1985) as pointed out by Vitale (1996). A more elementary proof of (1.1) based on the Prékopa-Leindler inequality and a result on "rounding" of a convex body due to Hadwiger is given in Vitale (1999). Moreover, Vitale (1996) proved that the inequality (1.1) gives a sharp right-tail probability bound of the random variable $\max _{1 \leq j \leq m} X_{j}$ and argued that the corresponding left-tail probability bound is not accessible from the methods of his paper.

Here among other things we will submit an alternative proof of (1.1) using a representation formula for Gaussian integrals discussed by Borell (2002) that has its origin in Fleming and Soner (1993). The key ingredient in the proof of this representation formula is a standard result in probability theory, namely the Girsanov theorem. Actually, our approach will lead to a slightly more general result than (1.1) so that a sharp left-tail probability bound of $\max _{1 \leq j \leq m} X_{j}$ becomes a corollary.

We will write $f \in \mathcal{K}$ if $f: \mathbf{R}^{m} \rightarrow \mathbf{R}$ is a Borel function such that $f\left(x_{1}, \ldots, x_{m}\right)$ is non-decreasing in each variable separately and

$$
f\left(x_{1}+s, \ldots, x_{m}+s\right) \leq f\left(x_{1}, \ldots, x_{m}\right)+s \text { if } x_{1}, \ldots, x_{m} \in \mathbf{R} \text { and } s \geq 0 .
$$

The following properties are immediate from the definition of the class $\mathcal{K}$ :

(a) $\mathcal{K}$ contains all constant functions.

(b) $\min _{1 \leq i \leq p} f_{i}, \max _{1 \leq i \leq p} f_{i} \in \mathcal{K}$ if $f_{1}, \ldots, f_{p} \in \mathcal{K}$.

(c) $\sum_{1}^{p} \alpha_{i} f_{i} \in \mathcal{K}$ if $f_{1}, \ldots, f_{p} \in \mathcal{K}, \alpha_{1}, \ldots, \alpha_{p} \geq 0$, and $\sum_{1}^{p} \alpha_{i} \leq 1$.

(d) $f \in \mathcal{K}$ if $f$ is smooth, $\partial f / \partial x_{j} \geq 0, j=1, \ldots, m$, and $\operatorname{div} f \leq 1$.

(e) If $f \in \mathcal{K}$ and $x, y=\left(y_{1}, \ldots, y_{m}\right) \in \mathbf{R}^{m}$, and $y_{j} \geq 0, j=1, \ldots, m$, then

$$
f(x+y) \leq f(y)+\max _{1 \leq j \leq m} y_{j} .
$$

(f) If $f \in \mathcal{K}$ and $x, y \in \mathbf{R}^{m}$,

$$
|f(x+y)-f(x)| \leq|y| \text {. }
$$


Here in Property (f) $|y|$ denotes the Euclidean norm of $y$. Stated otherwise Property (f) means that $f$ is Lipschitz continuous with Lipschitz constant one.

Theorem 1.1. Suppose $f \in \mathcal{K}$. Then $f(X) \in L^{1}(P)$ and

$$
E\left[\exp \left(f\left(X_{1}-\frac{\sigma_{1}^{2}}{2}, \ldots, X_{m}-\frac{\sigma_{m}^{2}}{2}\right)\right)\right] \leq \exp (E[f(X)]) .
$$

Moreover,

$$
E\left[\exp \left(f\left(X_{1}-\frac{\sigma_{1}^{2}}{2}, \ldots, X_{m}-\frac{\sigma_{m}^{2}}{2}\right)\right)\right] \geq e^{-\frac{\sigma_{\max }^{2}}{2}} \exp (E[f(X)])
$$

where $\sigma_{\max }=\max _{1 \leq j \leq m} \sigma_{j}$.

By choosing $f\left(x_{1}, \ldots, x_{m}\right)=\max _{1 \leq j \leq m} x_{j}$ in (1.3) we obtain the inequality (1.1).

Corollary 1.2. If $f \in \mathcal{K}$ is positively homogeneous of degree one, then

$$
P[f(X)-E[f(X)] \geq a] \leq \exp \left(-\frac{a^{2}}{2 \sigma_{\max }^{2}}\right)
$$

where $a>0$.

Since $X$ and $-X$ have the same probability law the inequality (1.5) with $f(X)=\min _{1 \leq j \leq m} X_{j}$ yields

$$
P\left[\max _{1 \leq j \leq m} X_{j}-E\left[\max _{1 \leq j \leq m} X_{j}\right] \leq-a\right] \leq \exp \left(-\frac{a^{2}}{2 \sigma_{\max }^{2}}\right) \text { if } a>0
$$

an inequality which, as mentioned above, seems impossible to deduce from (1.1) (cf Remark 2 in Vitale (1996)).

Using methods from diffusion theory, Ibragimov, Sudakov, and Tsirel'son (1976) derived the inequality (1.5) for $X_{1}, \ldots, X_{m} \in N(0,1)$ independent and 
$f$ a Lipschitz continuous function with Lipschitz constant one, a result which does not seem accessible by the appoach in this paper. Note that the inequality (1.3) is not true in this case as is readily seen by choosing $m=1$ and $f(x)=x^{-}, x \in \mathbf{R}$.

It is not obvious that the inequality (1.3) follows from Brunn-Minkowski theory as is the case with (1.1).

The paper is organized as follows. Section 1 recalls a result from stochastic analysis and Sections 3-4 are devoted to proofs of Theorem 1.1 and Corollary 1.2. Finally, in Section 5 Teorem 1.1 is used to compare certain option prices in the Black-Scholes and Bachelier models.

\section{A representation formula of Gaussian integrals}

Let $\gamma$ be the standard Gaussian measure on $\mathbf{R}^{n}$, that is

$$
d \gamma(x)=\exp \left(-\frac{|x|^{2}}{2}\right) \frac{d x}{\sqrt{2 \pi}^{n}}
$$

Here if $W=(W(t))_{t \geq 0}$ denotes a standard Brownian motion in $\mathbf{R}^{n}$, the law of $W(1)$ equals $\gamma$. For simplicity we think of $W$ as the identity map on the Fréchet space $C\left(\left[0, \infty\left[, \mathbf{R}^{n}\right)\right.\right.$ and $P$ stands for Wiener measure on this space. Furthermore $\mathcal{U}$ denotes the class of all $\mathbf{R}^{n}$-valued, bounded, and progressively measurable processes $u(t), t \geq 0$.

Now suppose $g: \mathbf{R}^{n} \rightarrow \mathbf{R}$ is a bounded Borel function. Then

$$
\int_{\mathbf{R}^{n}} e^{g} d \gamma=\exp \left(\sup _{u \in \mathcal{U}} E\left[g\left(W(1)+\int_{0}^{1} u(t) d t\right)-\frac{1}{2} \int_{0}^{1}|u(t)|^{2} d t\right]\right) .
$$

The formula in (2.1) originates from optimal control theory, see Remark 2.1 , pp. 257-58, in Fleming and Soner (1993). The present formulation is as in Borell (2002), where also a complete proof is given (see also Borell (2000)). As the proof of (1.1) in Vitale (1999) depends on the PrékopaLeinder inequality, it should be remarked that this inequality is an immediate consequence of (2.1), see Theorem 6.2 in Borell (2002). 
If $g: \mathbf{R}^{n} \rightarrow \mathbf{R}$ is a Borel function bounded from below, then by (2.1) and monotone convergence

$$
\int_{\mathbf{R}^{n}} e^{g} d \gamma \leq \exp \left(\sup _{u \in \mathcal{U}} E\left[g\left(W(1)+\int_{0}^{1} u(t) d t\right)-\frac{1}{2} \int_{0}^{1}|u(t)|^{2} d t\right]\right) .
$$

\section{Proof of Theorem 1.1}

Setting $\|x\|_{\infty}=\max _{1 \leq j \leq m}\left|x_{j}\right|$, Property (e) in Section 1 implies that

$$
f\left(x_{1}, \ldots, x_{m}\right) \leq f\left(\|x\|_{\infty}, \ldots,\|x\|_{\infty}\right) \leq f(0)+\|x\|_{\infty}
$$

and

$$
f\left(x_{1}, \ldots, x_{m}\right) \geq f\left(-\|x\|_{\infty}, \ldots,-\|x\|_{\infty}\right) \geq f(0)-\|x\|_{\infty}
$$

and, hence $f(X) \in L^{1}(P)$. Moreover, if $k \in \mathbf{N}_{+}$, Properties (a) and (b) in Section 1 show that $f_{k}=\max (-k, f) \in \mathcal{K}$. Thus proving (1.3), by Fatou's lemma and dominated convergence, there is no loss of generality in assuming that $f$ is bounded from below.

We proceed the proof of (1.3) by choosing an $m$ by $n$ matrice $A=$ $\left(a_{j k}\right)_{1 \leq j \leq m, 1 \leq k \leq n}$ with real entries such that the random vectors $X$ and $A W(1)$ possess the same probability law (here elements in $\mathbf{R}^{n}$ are identified with matrices of order $1 \times n)$. Now, if $a_{j}$ denotes the $j: t h$ row of $A$, by (2.2)

$$
\begin{aligned}
& E\left[\exp \left(f\left(X_{1}-\frac{\sigma_{1}^{2}}{2}, \ldots, X_{m}-\frac{\sigma_{m}^{2}}{2}\right)\right)\right] \\
\leq & \exp \left(\sup _{u \in \mathcal{U}} E\left[f\left(\left(a_{j} W(1)+a_{j} \int_{0}^{1} u(t) d t-\frac{\left|a_{j}\right|^{2}}{2}\right)_{j=1}^{m}\right)-\frac{1}{2} \int_{0}^{1}|u(t)|^{2} d t\right]\right) .
\end{aligned}
$$

Here for each $1 \leq j \leq m$,

$$
\begin{aligned}
& a_{j} \int_{0}^{1} u(t) d t=\int_{0}^{1} a_{j} u(t) d t \\
& \leq \int_{0}^{1} \frac{1}{2}\left(\left|a_{j}\right|^{2}+|u(t)|^{2}\right) d t
\end{aligned}
$$




$$
=\frac{\left|a_{j}\right|^{2}}{2}+\frac{1}{2} \int_{0}^{1}|u(t)|^{2} d t
$$

and since $f \in \mathcal{K}$, we get

$$
\begin{gathered}
f\left(\left(a_{j} W(1)+a_{j} \int_{0}^{1} u(t) d t-\frac{\left|a_{j}\right|^{2}}{2}\right)_{j=1}^{m}\right)-\frac{1}{2} \int_{0}^{1}|u(t)|^{2} d t \\
\leq f(A W(1)) .
\end{gathered}
$$

From this and (3.1) the inequality (1.3) follows at once.

The inequality (1.4) follows from Property (e) in Section 1 and the Jensen inequality, which completes the proof of Theorem 1.1.

\section{Proof of Corollary 1.2}

The inequality (1.5) is a consequence of (1.3) and Markov's inequality and a proof follows the same line of reasoning as in Vitale (1996). For completeness all details are given here. To begin with we use (1.3) with $X$ replaced by $r X$, where $r$ is a positive constant to obtain

$$
E\left[\exp \left(f\left(r X_{1}-\frac{r^{2} \sigma_{1}^{2}}{2}, \ldots, r X_{m}-\frac{r^{2} \sigma_{m}^{2}}{2}\right)\right] \leq \exp (E[f(r X)])\right.
$$

and next apply Property (e) in Section 1 and the homogeneity of $f$ to get

$$
E\left[\exp \left(r f(X)-\frac{r^{2} \sigma_{\max }^{2}}{2}\right)\right] \leq \exp (E[r f(X)])
$$

Consequently,

$$
E[\exp (r(f(X)-E[f(X)]))] \leq \exp \left(\frac{r^{2} \sigma_{\max }^{2}}{2}\right)
$$

and given $a>0$, the Markov inequality gives

$$
\begin{aligned}
P[f(X)-E[f(X)] & \geq a] \leq e^{-r a} E[\exp (r(f(X)-E[f(X)]))] \\
\leq & \exp \left(-r a+\frac{r^{2} \sigma_{\max }^{2}}{2}\right) .
\end{aligned}
$$


Here, if $\sigma_{\max }^{2}=0,(1.5)$ is trivial and if $\sigma_{\max }^{2}>0$ we choose $r=a / \sigma_{\max }^{2}$ to get (1.5). This completes the proof of Corollary 1.2.

\section{Comparison of certain option prices in the Black-Scholes and Bachelier models}

We will finish this paper by giving an interpretation in option pricing of the inequalities stated in the Abstract. For a detailed description of the theory of options, see Delbaen and Schachermayer (2006).

Consider a capital market with $m+1$ asset price processes $\hat{S}_{0}, \ldots, \hat{S}_{m}$

in the time interval $[0, T]$. We suppose $\hat{S}_{0}(t)>0$ for each $t$ and choose $\hat{S}_{0}$ as a numéraire and define the discounted price processes $S_{k}=\hat{S}_{k} / \hat{S}_{0}$, $k=0,1, \ldots, m$. Below we will consider two derivatives $\mathcal{D}_{\max }$ and $\mathcal{D}_{\min }$ of European type paying the amounts $Y=\max _{1 \leq j \leq m}\left\{S_{j}(T) / S_{j}(0)\right\}$ and $Z=$ $\min _{1 \leq j \leq m}\left\{S_{j}(T) / S_{j}(0)\right\}$ (in units of the numéraire), respectively to their owners at time of maturity $T$.

As above $W$ denotes a standard Brownian motion in $\mathbf{R}^{n}$. In the BlackScholes model,

$$
d S_{j}(t)=S_{j}(t) \delta_{j} d W(t), j=1, \ldots, m
$$

where $D=\left(\delta_{j k}\right)_{1 \leq j \leq m, 1 \leq k \leq n}$ is an appropriate volatility matrice with constant entries and the $j: t h$ row of $D$ is denoted by $\delta_{j}$. The prices of the derivatives $\mathcal{D}_{\text {max }}$ and $\mathcal{D}_{\text {min }}$ at time zero in this model equal

$$
\Pi_{Y}^{B S}=E\left[\max _{1 \leq j \leq m}\left\{\exp \left(\delta_{j} W(T)-\frac{\sigma_{j}^{2} T}{2}\right)\right\}\right]
$$

and

$$
\Pi_{Z}^{B S}=E\left[\min _{1 \leq j \leq m}\left\{\exp \left(\delta_{j} W(T)-\frac{\sigma_{j}^{2} T}{2}\right)\right\}\right]
$$

respectively, where $\sigma_{j}=\left|\delta_{j}\right|, j=1, \ldots, m$ (here matrices of order $1 \times n$ are identified with elements in $\mathbf{R}^{n}$ ).

In the Bachelier theory assuming the same volatility matrice as above,

$$
d S_{j}(t)=S_{j}(0) \delta_{j} d W(t), j=1, \ldots, m
$$


and the prices of the derivatives $\mathcal{D}_{\text {max }}$ and $\mathcal{D}_{\text {min }}$ at time zero equal

$$
\Pi_{Y}^{B}=E\left[\max _{1 \leq j \leq m}\left\{1+\delta_{j} W(T)\right\}\right]=1+E\left[\max _{1 \leq j \leq m}\left\{\delta_{j} W(T)\right\}\right]
$$

and

$$
\Pi_{Z}^{B}=E\left[\min _{1 \leq j \leq m}\left\{1+\delta_{j} W(T)\right\}\right]=1+E\left[\min _{1 \leq j \leq m}\left\{\delta_{j} W(T)\right\}\right]
$$

respectively.

In the following, $\Phi(x)=P[W(1) \leq x], x \in \mathbf{R}$, and $\varphi=\Phi^{\prime}$. Moreover, set

$$
c_{m}=m \int_{-\infty}^{\infty} x \varphi(x) \Phi^{m-1}(x) d x .
$$

Note that if $G_{1}, \ldots, G_{m} \in N(0,1)$ are independent, then

$$
c_{m}=E\left[\max _{1 \leq j \leq m} G_{j}\right] \text {. }
$$

Theorem 5.1. Suppose the asset prices $S_{1}, \ldots, S_{m}$ are non-negatively correlated. Then

(a)

$$
e^{-\frac{\sigma_{\max }^{2} T}{2}} \leq \frac{\Pi_{Y}^{B S}}{\Pi_{Y}^{B}} \leq \frac{\exp \left(\sigma_{\max } \sqrt{T} c_{m}\right)}{1+\sigma_{\max } \sqrt{T} c_{m}}
$$

(b) if $\sigma_{\max } \sqrt{T} c_{m}<1$,

$$
e^{-\frac{\sigma_{\max }^{2} T}{2}} \leq \frac{\Pi_{Z}^{B S}}{\Pi_{Z}^{B}} \leq \frac{\exp \left(-\sigma_{\max } \sqrt{T} c_{m}\right)}{1-\sigma_{\max } \sqrt{T} c_{m}}
$$

Proof. (a): By (1.1),

$$
\Pi_{Y}^{B S} \leq \exp \left(E\left[\max _{1 \leq j \leq m}\left\{\delta_{j} W(T)\right\}\right]\right)
$$

Moreover, the inequality (1.4) yields the lower bound

$$
\Pi_{Y}^{B S} \geq e^{-\frac{\sigma_{\max }^{2} T}{2}} \exp \left(E\left[\max _{1 \leq j \leq m}\left\{\delta_{j} W(T)\right\}\right]\right) .
$$


Thus

$$
e^{-\frac{\sigma_{\max T}^{2}}{2}} \leq \frac{\Pi_{Y}^{B S}}{\exp \left(\Pi_{Y}^{B}-1\right)} \leq 1
$$

and, since $\exp (x) \geq 1+x$ if $x \in \mathbf{R}$, we get

$$
e^{-\frac{\sigma_{\max }^{2} T}{2}} \leq \frac{\Pi_{Y}^{B S}}{\Pi_{Y}^{B}} \leq \frac{\exp \left(\Pi_{Y}^{B}-1\right)}{\Pi_{Y}^{B}}
$$

Here the member in the right-hand side of (5.1) is a non-decreasing function of $\Pi_{Y}^{B}$ in the interval $\left[1, \infty\left[\right.\right.$. Moreover, if $\varepsilon_{j} \in \mathbf{R}^{n}, j=1, \ldots, m$, and

$$
\left|\delta_{j}-\delta_{k}\right| \leq\left|\varepsilon_{j}-\varepsilon_{k}\right|, j, k=1, \ldots, m
$$

then by a variant of the Slepian lemma

$$
E\left[\max _{1 \leq j \leq m}\left\{\delta_{j} W(T)\right\}\right] \leq E\left[\max _{1 \leq j \leq m}\left\{\varepsilon_{j} W(T)\right\}\right]
$$

(see Theorem 3.15 in Ledoux and Talagrand (1991)). Clearly, there is no loss of generality in assuming $n \geq m$ and choosing $\varepsilon_{j}=\sigma_{\max } \sqrt{T} e_{j}, j=1, \ldots, m$, where $e_{1}, \ldots, e_{n}$ is the standard basis in $\mathbf{R}^{n}$, we have

$$
E\left[\max _{1 \leq j \leq m}\left\{\delta_{j} W(T)\right\}\right] \leq \sigma_{\max } \sqrt{T} c_{m}
$$

and Part (a) follows.

(b): As in the proof of Part (a) we get

$$
e^{-\frac{\sigma_{\max }^{2} T}{2}} \leq \frac{\Pi_{Z}^{B S}}{\exp \left(\Pi_{Z}^{B}-1\right)} \leq 1
$$

Therefore, if $\Pi_{Z}^{B}>0$,

$$
e^{-\frac{\sigma_{\max }^{2} T}{2}} \leq \frac{\Pi_{Z}^{B S}}{\Pi_{Z}^{B}} \leq \frac{\exp \left(\Pi_{Z}^{B}-1\right)}{\Pi_{Z}^{B}}
$$

where the the member in the right-hand side of (5.2) is a non-increasing function of $\Pi_{Z}^{B}$ in the interval ]0,1]. Moreover, since

$$
E\left[\min _{1 \leq j \leq m}\left\{\delta_{j} W(T)\right\}\right]=-E\left[\max _{1 \leq j \leq m}\left\{\delta_{j}(-W(T))\right\}\right] \geq-\sigma_{\max } \sqrt{T} c_{m}
$$


Part (b) follows at once. This completes the proof of Theorem 5.1.

As a simple numerical example we consider seven non-negatively correlated asset prices with yearly volatilities not exceeding 25 percent and the derivatives above with time to maturity at most 2 months i.e. $2 / 12$ years. Then Theorem 5.1 gives that $0.9948 \leq \Pi_{Y}^{B S} / \Pi_{Y}^{B} \leq 1.0088$ and $0.9948 \leq \Pi_{Z}^{B S} / \Pi_{Z}^{B} \leq 1.0106$. If we know that the yearly volatilities are less than 2.5 percent and all other assumptions are unchanged, then the difference between the upper and lower bound of the quotient $\Pi_{Y}^{B S} / \Pi_{Y}^{B}\left(\Pi_{Z}^{B S} / \Pi_{Z}^{B}\right)$ given in Theorem 5.1(a) (Theorem 5.1(b)) does not exceed 1.5 $\times 10^{-4}\left(1.5 \times 10^{-4}\right)$.

For the data reported by Bachelier in his thesis the yearly volatility was about 2.4 percent (see Schachermayer and Teichmann (2006)). Compare also Proposition 2 in Schachermayer and Teichmann (2006) which shows that the difference between at the money call prices in the Bachelier and Black-Scholes models is non-positive and of the order $O\left(\left(\sigma^{2} T\right)^{3 / 2}\right)$, where the volatility $\sigma$ of the underlying asset is assumed to be the same in the two

models. Furthermore, the same proposition ensures that the corresponding implied volatilities in the two models are very close.

\section{References}

Borell, Ch., 2000. Diffusion equations and geometric inequalities. Potential Analysis 12, 49-71.

Borell, Ch., 2002. Isoperimetry, log-concavity, and elasticity of option prices. In New Directions in Mathematical Finance (Wilmott, P., Rasmussen, H., eds), Wiley, pp. 73-91.

Delbaern, F., Schachermayer, W., 2006. The Mathematics of Arbitrage. Springer. 
Fleming, W.H., Soner, H.M., 1993. Controlled Markov Processes and Viscosity Solutions. Springer.

Ibragimov, I.A., Sudakov, V.N., Tsirel'son, B.S., 1976. Norms of Gaussian sample functions. Proceeding of the Third Japan-USSR Symposium on Probability. Lecture Notes in Math., Springer., 550, 20-21.

Ledoux, M., Talagrand, M., 1991. Probability in Banach Spaces. Isoperimetry and Processes. Springer.

Schachermayer, W., Teichmann, J., 2006. How close are the option pricing formulas of Bachelier and Black-Merton-Scholes? Math. Finance (to appear)

Tsirel'son, B.S., 1985. A geometric approach to maximum likelihood estimation for infinite-dimensional Gaussian location. II Theory Probab. Appl. $30,820-828$.

Vitale R.A., 1996. The Wills functional and Gaussian processes. Ann. Prob. 24, 2172-2178.

Vitale R.A., 1999. A log-concavity proof for a Gaussian exponential bound. In Advances in Stochastic Inequalities (Hill, T.P., Houdré, C., eds), Contemp. Math., AMS, 234, 209-212. . 